Bull. Austral. Math. Soc.

Vol. 50 (1994) [123-134]

\title{
SECOND-ORDER NORMAL VECTORS TO A CONVEX EPIGRAPH
}

\author{
Alberto Seeger
}

\begin{abstract}
The second-order behaviour of a nonsmooth convex function $f$ is reflected by the so-called second-order subdifferential mapping $\partial^{2} f$. This mathematical object has been intensively studied in recent years. Here we study $\partial^{2} f$ in connection with the geometric concept of "second-order normal vector" to the epigraph of $f$.
\end{abstract}

\section{MATHEMATICAL BACKGROUND}

Throughout this note $f: \mathbb{R}^{n} \rightarrow \mathbb{R} \cup\{+\infty\}$ is assumed to be a lower-semicontinuous proper convex function. As usual, the class of such functions is denoted by $\Gamma_{0}\left(\mathbb{R}^{n}\right)$. The purpose of this work is to provide the reader with some additional mathematical tools for a better understanding of the second-order behaviour of $f$ around a reference point $x \in \mathbb{R}^{n}$. Recall that the first-order behaviour of $f$ around $x$ is reflected by the set

$$
\partial f(x):=\left\{y \in \mathbb{R}^{n}: f\left(x^{\prime}\right) \geqslant f(x)+\left\langle y, x^{\prime}-x\right\rangle \text { for all } x^{\prime} \in \mathbb{R}^{n}\right\},
$$

where $\langle\cdot, \cdot\rangle$ stands for the usual Euclidean product in the space $\mathbb{R}^{n}$. The set (1.1) is known as the subdifferential of $f$ at $x$, and each of its elements is called a subgradient of $f$ at $x$ (see [6]).

Second-order information on $f$ is captured by a family of sets

$$
\left\{\partial^{2} f[x, y]: y \in \partial f(x)\right\} \text {. }
$$

The precise definition of $\partial^{2} f[x, y]$, and some new results concerning this set, will be given in Section 3 .

Twice epi-differentiability is a fundamental concept in the definition of $\partial^{2} f[x, y]$. A new characterisation of this notion will be given in Section 2 .

For convenience in our exposition, we recall below the concept of epigraphical convergence.

Received 27th October, 1993

Copyright Clearance Centre, Inc. Serial-fee code: 0004-9729/94 \$A2.00+0.00. 
Definition 1.1: (see, for instance, Attouch [1]). A sequence $\left\{\varphi_{k}\right\}_{k \in \mathbb{N}}$ of functions $\varphi_{k}: \mathbb{R}^{n} \rightarrow \overline{\mathbb{R}}$ is said to be epi-convergent to $\varphi: \mathbb{R}^{n} \rightarrow \overline{\mathbb{R}}$ if for every $h \in \mathbb{R}^{n}$, the following properties are satisfied:

$\exists\left\{h_{k}\right\} \rightarrow h$ such that $\varphi(h) \geqslant \lim \sup \varphi_{k}\left(h_{k}\right) ;$

$\forall\left\{h_{k}\right\} \rightarrow h$ one has $\varphi(h) \leqslant \liminf \varphi_{k}\left(h_{k}\right)$.

A family $\left\{\varphi_{t}\right\}_{t>0}$ of functions $\varphi_{t}: \mathbb{R}^{n} \rightarrow \overline{\mathbb{R}}$ epiconverges to $\varphi: \mathbb{R}^{n} \rightarrow \overline{\mathbb{R}}$ (as $t$ goes to $0^{+}$), if for all $\left\{t_{k}\right\} \rightarrow 0^{+}$, the sequence $\left\{\varphi_{t_{k}}\right\}$ epi-converges to $\varphi$. In such a case one says that $\varphi$ is the epigraphical limit of the family $\left\{\varphi_{t}\right\}_{t>0}$, and one writes $\varphi=$ epi- $\lim _{t \rightarrow 0^{+}} \varphi_{t}$.

\section{On Twice Epi-Differentiability}

In connection with the second-order analysis of nonsmooth functions, Rockafellar's concept of twice epi-differentiability has drawn the attention of many authors. In the case of nonsmoooth convex functions, this notion can be introduced in the following terms:

Definition 2.1: Let $f \in \Gamma_{0}\left(\mathbb{R}^{n}\right)$ be finite at $x$, and let $y \in \partial f(x)$. The function $f$ is said to be twice epi-differentiable at $x$ relative to $y$ if the epigraphical limit

$$
f^{\prime \prime}[x, y ; \cdot]:=\text { epi- } \lim _{t \rightarrow 0^{+}} \delta_{t}^{2} f[x, y ; \cdot]
$$

exists, where

$$
\delta_{t}^{2} f[x, y ; h]:=\frac{2}{t}\left[\frac{f(x+t h)-f(x)}{t}-\langle y, h\rangle\right] \text { for all } h \in \mathbb{R}^{n} .
$$

The function $f^{\prime \prime}[x, y ; \cdot]$ is called the second-order epi-derivative of $f$ at $x$ relative to $y$.

Important classes of convex functions enjoying the above twice epi-differentiability property have been singled out by Rockafellar [9] (see also [2, 8]). The existence of the second-order epi-derivative $f^{\prime \prime}[x, y ; \cdot]$ has been characterised in several equivalent ways by Moussaoui and Seeger [5]. These authors have shown that $\varepsilon$-subdifferentials, distance functions, and projections, are useful tools for studying this question. Here we follow another approach which consists in emphasising the role of the epigraph

$$
\text { epi } f:=\left\{(x, \beta) \in \mathbb{R}^{n} \times \mathbb{R}: f(x) \leqslant \beta\right\},
$$

or more precisely, of its indicator function

$$
\psi_{\text {epi } f}(x, \beta):=\left\{\begin{array}{ll}
0 & \text { if }(x, \beta) \in \text { epi } f \\
+\infty & \text { otherwise }
\end{array} .\right.
$$


A well-known fact in convex analysis is that

$$
y \in \partial f(x) \text { if and only if }(y,-1) \in N[f ; x] \text {, }
$$

where

$$
N[f ; x]:=\partial \psi_{\text {epi } f}(x, f(x))
$$

corresponds to the normal cone to epi $f$ at the point $(x, f(x))$. The equivalence (2.2) is sometimes expressed in the form

$$
\partial f(x)=\left\{y \in \mathbb{R}^{n}:(y,-1) \in N[f ; x]\right\}
$$

One of the main goals of this paper is to show that a somewhat similar formula also holds at a second-order level. This leads us to study the relationship between $f^{\prime \prime}\left[x, y_{i} \cdot\right]$ and $\psi_{\text {epi } f}^{\prime \prime}[(x, f(x)),(y,-1) ;(\cdot, \cdot)]$, the latter term being of course the second-order epi-derivative of $\psi_{\text {epi } f}$ at $(x, f(x))$ relative to $(y,-1)$. As a first step in our study, we look at the second-order differential quotients

$$
\varphi_{t}(h):=\delta_{t}^{2} f[x, y ; h]
$$

and

$$
\psi_{t}(h, \alpha):=\delta_{t}^{2} \psi_{\text {epi } f}[(x, f(x)),(y,-1) ;(h, \alpha)] .
$$

A simple matter of calculation yields:

LEMMA 2.1. Let $f \in \Gamma_{0}\left(\mathbb{R}^{n}\right)$ be finite at $x$, and let $y \in \partial f(x)$. Then, for all $t>0$ and $h \in \mathbb{R}^{n}$, one has

$$
\varphi_{t}(h)=\inf _{\alpha \in \mathbb{B}} \psi_{t}(h, \alpha)
$$

Moreover, if the function $f$ is finite at $x+t h$, then the infimum in (2.4) is attained at $\alpha=t^{-1}[f(x+t h)-f(x)]$.

Proof: By definition one has

$$
\psi_{t}(h, \alpha):=\frac{2}{t}\left[\frac{\psi_{\text {epi } f}((x, f(x))+t(h, \alpha))-\psi_{\text {epi } f}(x, f(x))}{t}-\langle(y,-1),(h, \alpha)\rangle\right] .
$$

After a short calculation one gets

$$
\psi_{t}(h, \alpha)=\frac{2}{t}\left[\psi_{\text {epi } f}(x+t h, f(x)+t \alpha)-\langle y, h\rangle+\alpha\right]
$$

that is to say,

$$
\psi_{t}(h, \alpha)= \begin{cases}\frac{2}{t}[\alpha-\langle y, h\rangle] & \text { if }(f(x+t h)-f(x)) / t \leqslant \alpha \\ +\infty & \text { otherwise }\end{cases}
$$


If $f$ is not finite at $x+t h$, then both terms in (2.4) are equal to $+\infty$. Otherwise, the function $\psi_{t}(h, \cdot)$ is minimised at $\alpha=t^{-1}[f(x+t h)-f(x)]$, and its infimum is just $\varphi_{t}(h)$.

Next we would like to pass to the limit as $t \rightarrow 0^{+}$in formula (2.4). An epigraphical limit is however a subtle concept, and needs to be handled with care. To avoid some undesirable technicalities, suppose that $x$ is a point at which the function $f \in \Gamma_{0}\left(\mathbb{R}^{n}\right)$ is continuous. This requirement is not too stringent and helps to keep our presentation clear. Under this continuity assumption, the directional derivative

$$
h \in \mathbb{R}^{n} \mapsto f^{\prime}(x ; h):=\lim _{t \rightarrow 0^{+}} t^{-1}[f(x+t h)-f(x)]
$$

is finite everywhere, and one has

$$
\lim t_{k}^{-1}\left[f\left(x+t_{k} h_{k}\right)-f(x)\right]=f^{\prime}(x ; h) \text { for all }\left\{\left(t_{k}, h_{k}\right)\right\} \rightarrow\left(0^{+}, h\right) .
$$

Now one can state the main result of this section.

Theorem 2.1. Let $f \in \Gamma_{0}\left(\mathbb{R}^{n}\right)$ be continuous at $x$, and let $y \in \partial f(x)$. Then the following statements are equivalent:

(a) $f$ is twice epi-differentiable at $x$ relative to $y$;

(b) $\psi_{\text {epi } f}$ is twice epi-differentiable at $(x, f(x))$ relative to $(y,-1)$.

For convenience, we split the proof of the above theorem into two lemmas.

Lemma 2.2. Let $f \in \Gamma_{0}\left(\mathbb{R}^{n}\right)$ be continuous at $x$, and let $y \in \partial f(x)$. Suppose $\psi_{\text {epi } f}$ is twice epi-differentiable at $(x, f(x))$ relative to $(y,-1)$. Then, $f$ is twice epi-differentiable at $x$ relative to $y$. Moreover, for all $h \in \mathbb{R}^{n}$, one can write

$$
\begin{aligned}
f^{\prime \prime}[x, y ; h] & =\inf _{\alpha \in \mathbb{B}} \psi_{\text {epi } f}^{\prime \prime}[(x, f(x)),(y,-1) ;(h, \alpha)] \\
& =\psi_{\text {epi } f}^{\prime \prime}\left[(x, f(x)),(y,-1) ;\left(h, f^{\prime}(x ; h)\right)\right] .
\end{aligned}
$$

Proof: Take any $(h, \alpha) \in \mathbb{R}^{n} \times \mathbb{R}$, and write

$$
\psi(h, \alpha):=\psi_{\text {epi } f}^{\prime \prime}[(x, f(x)),(y,-1) ;(h, \alpha)]=\left[\text { epi- } \lim _{t \rightarrow 0^{+}} \psi_{t}\right](h, \alpha) .
$$

If $\alpha<f^{\prime}(x ; h)$, then

$$
t^{-1}\left[f\left(x+t h^{\prime}\right)-f(x)\right]>\alpha^{\prime}
$$

for all $\left(t, h^{\prime}, \alpha^{\prime}\right)$ close to $\left(0^{+}, h, \alpha\right)$. This fact, together with expression (2.5), implies that

$$
\liminf _{\left(t, h^{\prime}, \alpha^{\prime}\right) \rightarrow\left(0^{+}, h, \alpha\right)} \psi_{t}\left(h^{\prime}, \alpha^{\prime}\right)=+\infty
$$


Thus, $\psi(h, \alpha)=+\infty$. Consider now the case $\alpha>f^{\prime}(x ; h)$. Since $y \in \partial f(x)$, one has necessarily $f^{\prime}(x ; h) \geqslant\langle y, h\rangle$. Hence, $\alpha-\langle y, h\rangle$ is strictly positive, and the term

$$
\frac{2}{t}\left[\alpha^{\prime}-\left\langle y, h^{\prime}\right\rangle\right]
$$

goes to $+\infty$ as $\left(t, h^{\prime}, \alpha^{\prime}\right)$ goes to $\left(0^{+}, h, \alpha\right)$. Thus, we are again in the situation described by (2.7). Summarising, $\psi(h, \alpha)=+\infty$ whenever $\alpha \neq f^{\prime}(x ; h)$. This implies of course that

$$
\inf _{\alpha \in \mathbb{R}} \psi(h, \alpha)=\psi\left(h, f^{\prime}(x ; h)\right) .
$$

It remains now to show that the function

$$
h \in \mathbb{R}^{n} \mapsto \psi\left(h, f^{\prime}(x ; h)\right)
$$

is the epigraphical limit of the family $\left\{\varphi_{t}\right\}_{t>0}$ as $t \rightarrow 0^{+}$. Take any sequence $\left\{t_{k}\right\} \rightarrow 0^{+}$ and any $h \in \mathbb{R}^{n}$. One needs to prove the conditions

$$
\exists\left\{h_{k}\right\} \rightarrow h \text { such that } \psi\left(h, f^{\prime}(x ; h)\right) \geqslant \limsup \varphi_{t_{k}}\left(h_{k}\right),
$$

and

$$
\forall\left\{h_{k}\right\} \rightarrow h \text { one has } \psi\left(h, f^{\prime}(x ; h)\right) \leqslant \liminf \varphi_{t_{k}}\left(h_{k}\right) .
$$

Since the epigraphical limit $\psi$ exists, one has

$$
\psi\left(h, f^{\prime}(x ; h)\right) \leqslant \liminf \psi_{t_{k}}\left(h_{k}, \alpha_{k}\right)
$$

for all $\left\{\left(h_{k}, \alpha_{k}\right)\right\} \rightarrow\left(h, f^{\prime}(x ; h)\right)$. But, for the particular choice

$$
\alpha_{k}=t_{k}^{-1}\left[f\left(x+t_{k} h_{k}\right)-f(x)\right]
$$

one gets

$$
\psi_{t_{k}}\left(h_{k}, \alpha_{k}\right)=\varphi_{t_{k}}\left(h_{k}\right)
$$

[See Lemma 2.1.]

Condition (2.9) is proven in this way. To prove (2.8) we use again the existence of the epigraphical limit $\psi$. One knows that

$$
\psi\left(h, f^{\prime}(x ; h)\right) \geqslant \lim \sup \psi_{t_{k}}\left(h_{k}, \alpha_{k}\right)
$$

for some $\left\{\left(h_{k}, \alpha_{k}\right)\right\} \rightarrow\left(h, f^{\prime}(x ; h)\right)$. If $\psi\left(h, f^{\prime}(x ; h)\right)=+\infty$, then condition (2.8) holds trivially. So, one can suppose that $\psi\left(h, f^{\prime}(x ; h)\right)<+\infty$, and

$$
\alpha_{k} \geqslant \beta_{k}:=t_{k}^{-1}\left[f\left(x+t_{k} h_{k}\right)-f(x)\right] .
$$


Now, notice that

$$
\psi_{t_{k}}\left(h_{k}, \alpha_{k}\right) \geqslant \psi_{t_{k}}\left(h_{k}, \beta_{k}\right)=\varphi_{t_{k}}\left(h_{k}\right)
$$

and hence

$$
\lim \sup \psi_{t_{k}}\left(h_{k}, \alpha_{k}\right) \geqslant \lim \sup \varphi_{t_{k}}\left(h_{k}\right) .
$$

Condition (2.8) follows by combining (2.11) and the above inequality.

Next we state the converse of Lemma 2.2 .

Lemma 2.3. Let $f \in \Gamma_{0}\left(\mathbb{R}^{n}\right)$ be continuous at $x$, and let $y \in \partial f(x)$. Assume that $f$ is twice epi-differentiable at $x$ relative to $y$. Then, $\psi_{\text {epi } f}$ is twice epi-differentiable at $(x, f(x))$ relative to $(y,-1)$. Moreover, for all $(h, \alpha) \in \mathbb{R}^{n} \times \mathbb{R}$, one can write

$$
\psi_{\text {epi } f}^{\prime \prime}[(x, f(x)),(y,-1) ;(h, \alpha)]=\left\{\begin{array}{ll}
f^{\prime \prime}[x, y ; h] & \text { if } \alpha=f^{\prime}(x ; h) \\
+\infty & \text { otherwise }
\end{array} .\right.
$$

Proof: We keep the same notation as in the proof of the previous lemma. Take any sequence $\left\{t_{k}\right\} \rightarrow 0^{+}$and any $(h, \alpha) \in \mathbb{R}^{n} \times \mathbb{R}$. One has seen already that if $\alpha \neq$ $f^{\prime}(x ; h)$, then the second-order epi-derivative $\psi$ is well defined at $(h, \alpha)$, and it is equal to $+\infty$. So, we just need to consider the case $\alpha=f^{\prime}(x ; h)$, and prove the conditions

$$
\exists\left\{\left(h_{k}, \alpha_{k}\right)\right\} \rightarrow\left(h, f^{\prime}(x ; h)\right) \text { such that } f^{\prime \prime}[x, y ; h] \geqslant \limsup \psi_{t_{k}}\left(h_{k}, \alpha_{k}\right),
$$

and

$$
\forall\left\{\left(h_{k}, \alpha_{k}\right)\right\} \rightarrow\left(h, f^{\prime}(x ; h)\right) \text { one has } f^{\prime \prime}[x, y ; h] \leqslant \liminf \psi_{t_{k}}\left(h_{k}, \alpha_{k}\right) .
$$

But the first one follows from the existence of $f^{\prime \prime}[x, y ; h]$ and the possibility of choosing $\left\{\alpha_{k}\right\}$ as in (2.10). To prove the second condition, observe that

$$
\psi_{t_{k}}\left(h_{k}, \alpha_{k}\right) \geqslant \varphi_{t_{k}}\left(h_{k}\right)
$$

[see Lemma 2.1] no matter how one chooses the sequence $\left\{\alpha_{k}\right\}$. Thus

$$
f^{\prime \prime}[x, y ; h] \leqslant \liminf \varphi_{t_{k}}\left(h_{k}\right) \leqslant \liminf \psi_{t_{k}}\left(h_{k}, \alpha_{k}\right) .
$$

This completes the proof of the lemma.

Lemmas 2.2 and 2.3 not only serve to prove Theorem 2.1 , but also provide some formulae linking the second-order epi-derivatives $f^{\prime \prime}[x, y ; \cdot]$ and $\psi_{\text {epi } f}^{\prime \prime}[(x, f(x)),(y,-1)$; $(\cdot, \cdot)]$ in a simple way. These formulae have many interesting consequences, some of which will be explored in the next section. 


\section{On Second-Order Normal Directions}

As explained by the author in $[11,12,5]$, to each second-order epi-derivative $f^{\prime \prime}[x, y ; \cdot]$ one can associate a unique nonempty closed convex set $\partial^{2} f[x, y]$ in such a way that

$$
f^{\prime \prime}[x, y ; h]=\left[\sup \left\{\langle z, h\rangle: z \in \partial^{2} f[x, y]\right\}\right]^{2} \text { for all } h \in \mathbb{R}^{n} .
$$

More precisely:

Definition 3.1: Let $f, x$, and $y$, be as in Definition 2.1. The second-order subdifferential of $f$ at $x$ relative to $y$ is the set given by

$$
\partial^{2} f[x, y]:=\left\{z \in \mathbb{R}^{n}:\langle z, h\rangle \leqslant\left\{f^{\prime \prime}[x, y ; h]\right\}^{1 / 2} \text { for all } h \in \mathbb{R}^{n}\right\} .
$$

Each vector $z$ in $\partial^{2} f[x, y]$ is called a second-order subgradient of $f$ at $x$ relative to $y$.

REMARK 3.1. A variant of the set (3.1) is obtained by using pointwise convergence instead of epigraphical convergence (see $[3,4,10]$ ). However, such a variant is of less interest, at least in the context of this note.

Second-order normal directions to a given convex set are obtained by applying the concept of second-order subdifferentiability to its corresponding indicator function. In the specific case of a convex epigraph, one has:

Definition 3.2: Let $f \in \Gamma_{0}\left(\mathbb{R}^{n}\right)$ be finite at $x$, and let $y \in \partial f(x)$. If $\psi_{\text {epi } f}$ is twice epi-differentiable at $(x, f(x))$ relative to $(y,-1)$, then each vector in the set

$$
N^{2}[f ; x, y]:=\partial^{2} \psi_{\text {epi }}[((x, f(x)),(y,-1)]
$$

is called a second-order normal vector to epi $f$ at $(x, f(x))$ relative to $(y,-1)$.

An equivalent definition of the set $N^{2}[f ; x, y]$ can be found in our previous work [12]. The superscript 2 over the capital letter $N$ reminds us that we are working at a second-order level. $N^{2}[f ; x, y]$ is a closed convex set in $\mathbb{R}^{n} \times \mathbb{R}$ which contains the origin. However, this set is not always a cone.

The purpose of this section is to explore the connection existing between the second-order subgradients of a convex function, and the second-order normal vectors to its epigraph. As an extension of formula (2.3), one gets the following nice result:

THEOREM 3.1. Let $f \in \Gamma_{0}\left(\mathbb{R}^{n}\right)$ be continuous at $x$, and let $y \in \partial f(x)$. Assume any of the equivalent conditions in Theorem 2.1. Then,

$$
\partial^{2} f[x, y]=\left\{z \in \mathbb{R}^{n}:(z, 0) \in N^{2}[f ; x, y]\right\} .
$$

Proof: By definition, $\partial^{2} f[x, y]$ is the subdifferential at $0 \in \mathbb{R}^{n}$ of the sublinear function $q:=\left\{f^{\prime \prime}[x, y, \cdot]\right\}^{1 / 2}$. Similarly, $N^{2}[f ; x, y]$ is the subdifferential at 
$(0,0) \in \mathbb{R}^{n} \times \mathbb{R}$ of the sublinear function $\ell:=\left\{\psi_{\text {epi }}^{\prime \prime}[(x, f(x)),(y,-1) ;(\cdot, \cdot)]\right\}^{1 / 2}$. Now, according to Lemma 2.2 , one can write

$$
q(h)=\inf _{\alpha \in \mathbb{R}} \ell(h, \alpha) \quad \text { for all } h \in \mathbb{R}^{n} .
$$

Moreover, for $h=0$ the above infimum is attained at $\alpha=f^{\prime}(x ; 0)=0$. By applying Rockafellar's rule [7, Theorem 24] on the subdifferential of a marginal function, one gets

$$
\partial q(0)=\left\{z \in \mathbb{R}^{n}:(z, 0) \in \partial \ell(0,0)\right\} .
$$

But this is just another way of writing formula (3.3).

Theorem 3.1 says that $\partial^{2} f[x, y]$ can be identified with the section of $N^{2}[f ; x, y]$ corresponding to the height $\gamma=0$. Recall that for computing first-order subgradients one has to cut the normal cone $N[f ; x]$ at the level $\gamma=-1$. Below we illustrate this situation with the help of an example.

EXAMPLE 3.1. Let $f: \mathbb{R} \rightarrow \mathbb{R}$ be given by

$$
f(x)=\max \left\{\frac{1}{2}(x-1)^{2}, \frac{1}{2}(x+1)^{2}\right\} .
$$

For $x=0$, one has $N[f ; x]=\{(y, \gamma) \in \mathbb{R} \times \mathbb{R}:|y|+\gamma \leqslant 0\}$. By cutting this normal cone at the level $\gamma=-1$, one gets $\partial f(x)=\{y \in \mathbb{R}:|y|-1 \leqslant 0\}=[-1,1]$. Take, for instance, the subgradient $y=1$. As a matter of computation one gets $N^{2}[f ; x, y]=$ $\{(z, \gamma) \in \mathbb{R} \times \mathbb{R}: z+\gamma \leqslant 1\}$. The set $\partial^{2} f[x, y]$ is obtained by setting $\gamma=0$ in the inequality $z+\gamma \leqslant 1$. Thus, $\partial^{2} f[x, y]=\{z \in \mathbb{R}: z \leqslant 1\}=(-\infty, 1]$.

The next result is somehow the converse of Theorem 3.1. It tells us how to compute $N^{2}[f ; x, y]$ in terms of $\partial^{2} f[x, y]$.

TheOREM 3.2. Under the same assumptions as in Theorem 3.1, one can write

$$
N^{2}[f ; x, y]=\left\{(z, \gamma) \in \mathbb{R}^{n} \times \mathbb{R}: z+\gamma y \in \partial^{2} f[x, y]\right\} .
$$

Proof: By definition, $(z, \gamma) \in N^{2}[f ; x, y]$ if and only if

$$
\langle(z, \gamma),(h, \alpha)\rangle \leqslant\left\{\psi_{\text {epi } f}^{\prime \prime}[(x, f(x)),(y,-1) ;(h, \alpha)]\right\}^{1 / 2} \text { for all }(h, \alpha) \in \mathbb{R}^{n} \times \mathbb{R} .
$$

According to Lemma 2.3, the above condition reduces to

$$
\left\langle(z, \gamma),\left(h, f^{\prime}(x ; h)\right)\right\rangle \leqslant\left\{f^{\prime \prime}[x, y ; h]\right\}^{1 / 2} \text { for all } h \in \mathbb{R}^{n} .
$$

This is clearly equivalent to

$$
\langle z, h\rangle+\gamma f^{\prime}(x ; h) \leqslant\left\{f^{\prime \prime}[x, y ; h]\right\}^{1 / 2} \text { for all } h \in D
$$


where

$$
D:=\left\{h \in \mathbb{R}^{n}: f^{\prime \prime}[x, y ; h]<+\infty\right\}
$$

denotes the effective domain of $f^{\prime \prime}[x, y ; \cdot]$. But on the set $D$, the directional derivative $f^{\prime}(x ; \cdot)$ coincides with the linear function $\langle y, \cdot\rangle$. Thus, condition (3.5) can be written in the form

$$
\langle z+\gamma y, h\rangle \leqslant\left\{f^{\prime \prime}[x, y ; h]\right\}^{1 / 2} \text { for all } h \in D .
$$

The latter inequality amounts to saying that $z+\gamma y \in \partial^{2} f[x, y]$.

We mention that Theorem 3.2 yields a simple expression for the polar set of $N^{2}[f ; x, y]$ in terms of the polar set of $\partial^{2} f[x, y]$. Polarity is an interesting tool in the analysis of closed convex sets containing the origin. By definition, the polar set of $C \subset \mathbb{R}^{n}$ is given by

$$
C^{0}:=\left\{h \in \mathbb{R}^{n}:\langle z, h\rangle \leqslant 1 \text { for all } z \in C\right\}
$$

COROLlary 3.1. Under the same assumptions as in Theorem 3.1, the polar set of $N^{2}[f ; x, y]$ is given by

$$
\begin{aligned}
\left\{N^{2}[f ; x, y]\right\}^{0} & =\left\{(h,\langle y, h\rangle): h \in\left(\partial^{2} f[x, y]\right)^{0}\right\} \\
& =\left\{(h,\langle y, h\rangle): f^{\prime \prime}[x, y ; h] \leqslant 1\right\}
\end{aligned}
$$

Proof: Let $L: \mathbb{R}^{n} \times \mathbb{R} \rightarrow \mathbb{R}^{n}$ be the linear mapping given by

$$
L(z, \gamma)=z+\gamma y
$$

By applying Theorem 3.2 and a standard calculus rule on polar sets (see [6, Corollary 16.3.2]), one obtains

$$
\left\{N^{2}[f ; x, y]\right\}^{0}=\left\{L^{-1}\left(\partial^{2} f[x, y]\right)\right\}^{0}=L^{*}\left(\left\{\partial^{2} f[x, y]\right\}^{0}\right)
$$

where $L^{*}: \mathbb{R}^{n} \rightarrow \mathbb{R}^{n} \times \mathbb{R}$ stands for the adjoint mapping of $L$. It suffices now to observe that $L^{*}$ is given by $L^{*} h=(h,\langle y, h\rangle)$.

We end this section by mentioning another by-product of the formulae established in Lemmas 2.2 and 2.3. The next proposition deals with the second-order epi-derivative of the Legendre-Fenchel conjugate $f^{*} \in \Gamma_{0}\left(\mathbb{R}^{n}\right)$ of $f$. It has been proven by Rockafellar [9, Theorem 2.4] that the existence of $f^{\prime \prime}[x, y ; \cdot]$ is equivalent to the existence of $\left(f^{*}\right)^{\prime \prime}[y, x ; \cdot]$; moreover, both second-order epi-derivatives are related by the conjugacy relationship

$$
\frac{1}{2}\left(f^{*}\right)^{\prime \prime}[y, x ; z]=\left\{\frac{1}{2} f^{\prime \prime}[x, y ; \cdot]\right\}^{*}(z) \text { for all } z \in \mathbb{R}^{n}
$$


We show next that $\left(f^{*}\right)^{\prime \prime}[y, x ; \cdot]$ can also be expressed in terms of the second-order epi-derivative

$$
(z, \gamma) \in \mathbb{R}^{n} \times \mathbb{R} \mapsto \sigma_{\text {epi } f}^{\prime \prime}[(y,-1),(x, f(x)) ;(z, \gamma)]
$$

where

$$
\sigma_{\text {epi } f}:=\psi_{\text {epi } f}^{*}
$$

stands for the support function of epi $f$.

Proposition 3.1. Under the same assumptions as in Theorem 3.1, one can write

$$
\sigma_{\text {epi } f}^{\prime \prime}[(y,-1),(x, f(x)) ;(z, \gamma)]=\left(f^{*}\right)^{\prime \prime}[y, x ; z+\gamma y] \text { for all }(z, \gamma) \in \mathbb{R}^{n} \times \mathbb{R}
$$

In particular,

$$
\left(f^{*}\right)^{\prime \prime}[y, x ; z]=\sigma_{\text {epi } f}^{\prime \prime}[(y,-1),(x, f(x)) ;(z, 0)] \text { for all } z \in \mathbb{R}^{n} .
$$

Proof: According to Lemma 2.3, one has

$$
\frac{1}{2} \psi(h, \alpha)= \begin{cases}\frac{1}{2} f^{\prime \prime}[x, y ; h] & \text { if } \alpha=f^{\prime}(x ; h), \\ +\infty & \text { otherwise }\end{cases}
$$

where $\psi(h, \alpha):=\psi_{\text {epi } f}^{\prime \prime}[(x, f(x)),(y,-1) ;(h, \alpha)]$. By taking the Legendre-Fenchel conjugate on both sides of (3.8), one gets

$$
\left(\frac{1}{2} \psi\right)^{*}(z, \gamma)=\sup _{\substack{h \in \mathbb{R}^{n} \\ \alpha \in \mathbb{R}}}\left\{\langle z, h\rangle+\gamma \alpha-\frac{1}{2} f^{\prime \prime}[x, y ; h]: \alpha=f^{\prime}(x ; h)\right\}
$$

In the above supremum, one can let $h$ range over the effective domain $D$ of $f^{\prime \prime}[x, y ; \cdot]$. If $h$ belongs to such a set $D$, then $f^{\prime}(x ; h)=\langle y, h\rangle$. Hence,

$$
\begin{aligned}
\left(\frac{1}{2} \psi\right)^{*}(z, \gamma) & =\sup _{h \in D} \ell\left\{\langle z+\gamma y, h\rangle-\frac{1}{2} f^{\prime \prime}[x, y ; h]\right\} \\
& =\left\{\frac{1}{2} f^{\prime \prime}[x, y ; \cdot]\right\}^{*}(z+\gamma y)
\end{aligned}
$$

By using Rockafellar's conjugacy relationship [9, Theorem 2.4], one obtains finally

$$
\frac{1}{2} \sigma_{\text {epi } f}^{\prime \prime}[(y,-1),(x, f(x)) ;(z, \gamma)]=\frac{1}{2}\left(f^{*}\right)^{\prime \prime}[y, x ; z+\gamma y]
$$

The proof of the proposition is now complete. 


\section{Conclusions}

As one may expect, the epigraph epi $f$ carries in a hidden way information on the second-order behaviour of the convex function $f$. To bring this information into light, it suffices to collect all second-order normal vectors to epi $f$. Of course, one can localise this search around a reference point $x$, and a reference subgradient $y$. Once we have evaluated the set $N^{2}[f ; x, y]$, it is possible to get $\partial^{2} f[x, y]$ by using the cutting procedure explained in Theorem 3.1. If one wishes to move in the opposite direction, one can invoke Theorem 3.2. Indeed, formula (3.4) tells us how to construct $N^{2}[f ; x, y]$ starting from $\partial^{2} f[x, y]$.

UP to some minor modifications, the results presented in this note can be extended to an infinite dimensional setting. For instance, on a reflexive Banach space, the symbol $\langle\cdot, \cdot\rangle$ has to be understood as a duality product, epigraphical convergence has to be changed by Mosco-convergence, and so on.

\section{REFERENCES}

[1] H. Attouch, Variational convergence for functions and operators, Applied Math. Series (Pitman, 1984).

[2] R. Cominetti, 'On pseudo-differentiability', Trans. Amer. Math. Soc. 324 (1991), 843-865.

[3] J.-B. Hiriart-Urruty, 'A new set-valued second-order derivative for convex functions', in Mathematics for optimization, (J.-B. Hiriart-Urruty, Editor) (North-Holland, 1986).

[4] J.-B. Hiriart-Urruty and A. Seeger, 'Calculus rules on a new set-valued second-order derivative for convex functions', Nonlinear Anal. 13 (1989), 721-738.

[5] M. Moussaoui and A. Seeger, 'Second-order subgradients of convex integral functionals', preprint, Department of Mathematics, University of Avignon, July 1993.

[6] R.T. Rockafellar, Convex analysis (Princeton Univ. Press, Princeton, 1970).

[7] R.T. Rockafellar, Conjugate duality and optimization, Conference Board of Math. Sci. Series No. 16 (SIAM Publications, 1974).

[8] R.T. Rockafellar, 'First and second order epi-differentiability in nonlinear programming', Trans. Amer. Math. Soc. 307 (1988), 75-108.

[9] R.T. Rockafellar, 'Generalized second derivatives of convex functions and saddle functions', Trans. Amer. Math. Soc. 322 (1990), 810-822.

[10] A. Seeger, Analyse du second ordre de problèmes non différentiables, Ph.D. Thesis (Université Paul Sabatier, Toulouse, 1986).

[11] A. Seeger, 'Second derivatives of a convex function and of its Legendre-Fenchel transformate', SIAM J. Optim. 2 (1992), 405-424.

[12] A. Seeger, 'Limiting behavior of the approximate second-order subdifferential of a convex function', J. Optim. Theory Appl. 74 (1992), 527-544. 
King Fahd University of Petroleum and Minerals Department of Mathematical Sciences

Dhahran 31261

Saudi Arabia 